\title{
La voie à suivre pour la promotion de la santé et la prévention
}

\section{Christine Romann ${ }^{a}$, Barbara Weil ${ }^{b}$}

a Membre du Comité central de la FMH, responsable du domaine Promotion de la santé et prévention

b Responsable du département Promotion de la santé et prévention de la FMH
Correspondance:

Dr Christine Romann

$\mathrm{FMH}$

Elfenstrasse 18

CH-3000 Berne 15

Tél. 0313591111

Fax 0313591112
Grâce à sa nouvelle stratégie en matière de promotion de la santé et de prévention, la FMH entend apporter une contribution décisive pour une Suisse en meilleure santé d'ici à 2020 - tel est l'objectif que nous nous fixons. A cette fin, la FMH veut assumer un rôle clé et clarifier et répartir les tâches nécessaires dans ses propres rangs. Convaincu du bien-fondé de la promotion de la santé et de la prévention, le corps médical fait bénéficier de ses compétences tous les domaines où il est possible d'agir; les médecins participent activement à l'élaboration et à la mise en ouvre des programmes spécifiques.

Voici, dans les grandes lignes, la nouvelle stratégie que le domaine Promotion de la santé et prévention de la FMH soumettra à l'Assemblée des délégués pour discussion et approbation en septembre.

Jetons un bref regard en arrière: au cours des années 80 déjà, la FMH a reconnu l'importance de la prévention en matière de $\mathrm{VIH}$ ou de désaccoutumance au tabac. Elle a participé aux débats sur la politique en matière de toxicomanie et a élaboré un concept sur la drogue qui n'a rien perdu de sa pertinence. Elle a mis sur pied la commission consultative «Prévention et promotion de la santé», intitulée aujourd'hui «Commission de prévention». Au début des années 90, le département Prévention a finalement vu le jour. Sa principale tâche consistait à concevoir des programmes de formation continue en collaboration avec l'OFSP qui organisait à l'époque une campagne de prévention tous les 3 à 4 ans sur un sujet spécifique. D'aucuns se souviendront sans doute des sessions de formation continue «Crise et suicide» ou «Back in Time», un projet sur la détection précoce et une meilleure prise en charge des lombalgies. Après que le Comité central a décidé, au début de l'année 2000, de se consacrer davantage aux tâches stratégiques, le département est devenu au fil des ans un centre de compétences pour toutes les questions de politique de la santé en matière de prévention. Petit à petit, la FMH a siégé dans les principaux organes nationaux et a eu son mot à dire dans les procédures de consultation les plus importantes. Aujourd'hui, elle est un partenaire incontournable pour toutes les questions relatives à la promotion de la santé et la prévention.

A l'heure actuelle, la santé fait partie des préoccupations majeures, tant pour les individus que pour la société en général. Il n'est donc pas étonnant que ce sujet soit largement traité en politique, comme le montrent les discussions en cours au sujet de la loi sur la prévention. Nul ne conteste que l'on puisse et doive encore faire beaucoup pour promouvoir la santé et prévenir les maladies. Il est également attesté que la prévention s'avère financièrement rentable et permet de freiner l'augmentation constante des coûts. Cependant, si l'on applique sérieusement le principe de prévention, de gros intérêts commerciaux peuvent aussi entrer en jeu, il suffit de penser à la prévention du tabagisme. Alors que le Parlement est en pleine discussion pour savoir quel rôle l'Etat devra jouer en matière de prévention, la FMH a d'autres priorités: quelles seront ses principaux objectifs dans le domaine de la promotion de la santé et de la prévention? Avec quels partenaires collaborer pour atteindre l'objectif d'une population en meilleure santé? Comment répartir les tâches à l'interne? Quelles sont les questions qui se posent en matière de prévention et de promotion de la santé dans les différentes disciplines? Quels projets cantonaux ou communaux requièrent notre engagement et nos compétences médicales? Quelle place faut-il octroyer à la promotion de la santé et à la prévention dans notre pratique quotidienne? Comment indemniser notre travail dans ce domaine? Et last, but certainly not least, que signifie promotion de la santé et prévention pour nous médecins? Car notre propre santé nous tient aussi à cœur!

Nous nous réjouissons d'un débat animé avec toutes les personnes intéressées par la promotion de la santé et la prévention et espérons avoir mis sur de bons rails notre projet pour une Suisse en meilleure santé afin qu'il se poursuive et garde son cap au cours de ces prochains mois et des années à venir. 